\title{
An Empirical Study on Portfolio Risk Analysis Based on Copula - GARCH
}

\author{
Rui Wang ${ }^{1, a}$ \\ ${ }^{1}$ Department of Mathematics and Physics, University of Science and Technology Beijing \\ 30 Xueyuan Road, Beijing, 100083, China \\ aflantic_wr@163.com
}

Keywords: Copula-GARCH model, portfolio, Monte Carlo simulation, Risk Analysis.

\begin{abstract}
Based on Copula function and GARCH model, this paper establishes the Copula-GARCH-GED model to analyze the portfolio risk of four stocks in different industries-Minsheng Investment, Huayi Brothers, Renhe Pharmaceutical and Yanghe Shares in the Shenzhen stock market. And then, we use the Monte Carlo simulation method, in the case of different confidence coefficients and the minimum risk, to calculate the investment ratio of the four assets and obtain the portfolio of VaR.
\end{abstract}

\section{Introduction}

Risk management has always been an important subject in the field of financial research. With the tremendous changes of the international financial markets, financial risk is increasing continuously. Taking a variety of investment strategies to avoid risks has reached a consensus among investors. A large number of empirical studies have shown that choosing a combination of less relevant assets can reduce the risk of investment.

Copula is a statistical method used to describe the interdependent structure of multiple random variables, and risk management is an important application area of Copula theory [1]. The purpose of this paper is to use Copula-GARCH method to study the portfolio investment risk of multiple assets, estimate the risk of portfolio investment in the future, and give the specific combination form under the principle of risk minimization.

\section{Selection and Distribution of Sample Data}

The empirical data of this paper will be composed of four stocks belonging to different industries, such as Minsheng Investment (MI), Huayi Brothers (HB), Renhe Pharmaceutical (RP) and Yanghe Shares (YS). We select the daily closing price $\left(P_{t}\right)$ in 2014.1.1-2015.12.31 as the sample data, a total of 650 sets of data, and then take the $\log$ yield $R_{t}=100\left(\ln \left(P_{t}\right)-\ln \left(P_{t-1}\right)\right)$. The data comes from the RESSET financial research database [2].

Normality Test. Commonly used normality test is Jarque-Bera test. Under the normality assumption, the JB test is gradually obeying the $\chi^{2}$ distribution of degrees of freedom. Table 1 is the basic statistics on the yield of Minsheng Investment, Huayi Brothers, Renhe Pharmaceutical and Yanghe Shares:

Table 1 Basic Statistics of the Sample Data

\begin{tabular}{c|c|c|c|c}
\hline & MI & HB & RP & YS \\
\hline Mean & 0.6911 & -0.0641 & 0.1019 & 0.0604 \\
\hline Kurtosis & 11.15304 & 7.760888 & 17.27923 & 75.07423 \\
\hline Skewness & -0.395179 & 0.073373 & -1.304267 & -4.726716 \\
\hline J-B & 1814.4060 & 613.5103 & 5697.7010 & 142889.8 \\
\hline P-Value & 0.0000 & 0.0000 & 0.0000 & 0.0000 \\
\hline
\end{tabular}

From Table 1, we have that the kurtosis of the sample is greater than 3, and the absolute value of skewness is greater than 0 . The skewnesses of the MI, RP and YS are negative, indicating that the 
empirical distribution of the sample has a significant variance of variance and an obvious peak and fat tails compared with the corresponding normal distribution. So we reject the hypothesis that the yield is a normal distribution [3].

Stability Test. From Fig.1, we have that the yield fluctuates around the mean, which means that there is no obvious trend. Therefore, we need to do the ADF test for the simple data with the intercept term without the time trend item.
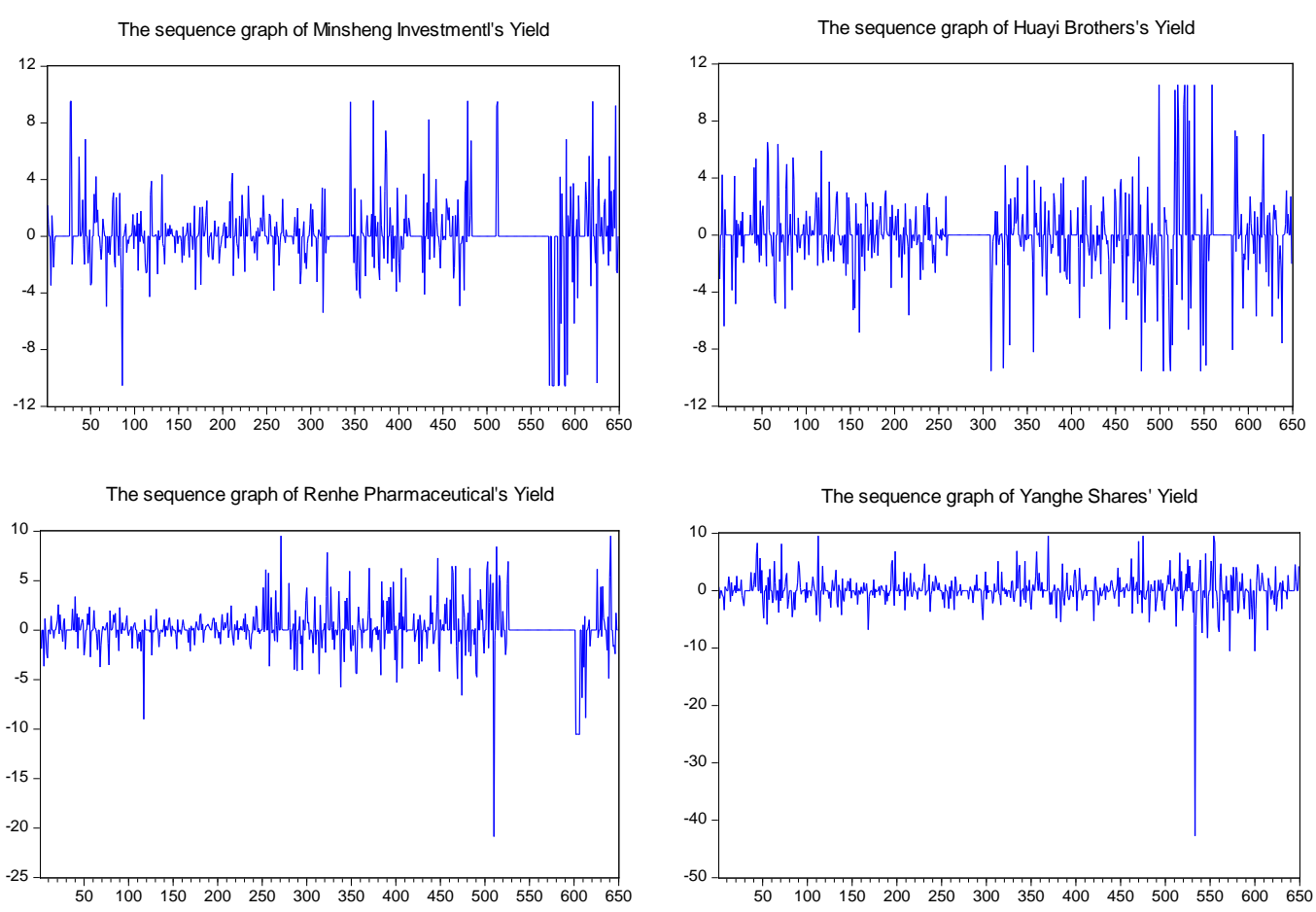

Fig.1 The sequence diagram of the yield of the sample data

Table 2 ADF Test of Sample Data

\begin{tabular}{c|c|c|c|c}
\hline & MI & HB & RP & YS \\
\hline ADF & -23.6367 & -24.4128 & -23.0606 & -24.0532 \\
\hline 1\% level & -3.4402 & -3.4402 & -3.4402 & -3.4402 \\
\hline 5\% level & -2.8658 & -2.8658 & -2.8658 & -2.8658 \\
\hline 10\% level & -2.5691 & -2.5691 & -2.5691 & -2.5691 \\
\hline
\end{tabular}

The data in Table 2 show that the ADF test values of the yield sequences are all less than 1\% corresponding to the critical value, so the original hypothesis is rejected. It can be said that the sequences of four stocks yields are stable, that is, the model can be modeled directly without distinction [4].

Correlation Analysis. Fig.2 - Fig.3 depict the autocorrelation and partial autocorrelation functions of four stocks:
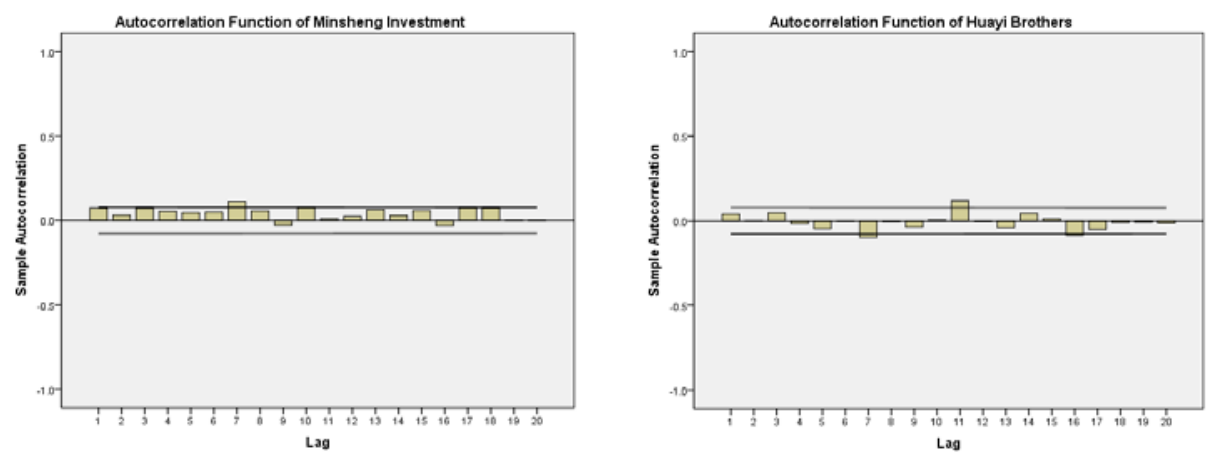

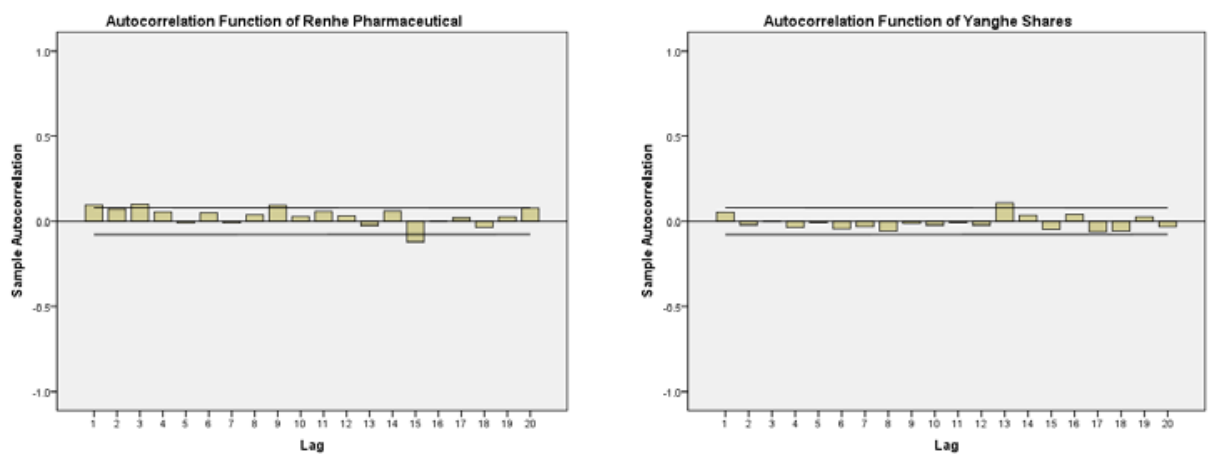

Fig.2 Autocorrelation Function Images of Sample Data
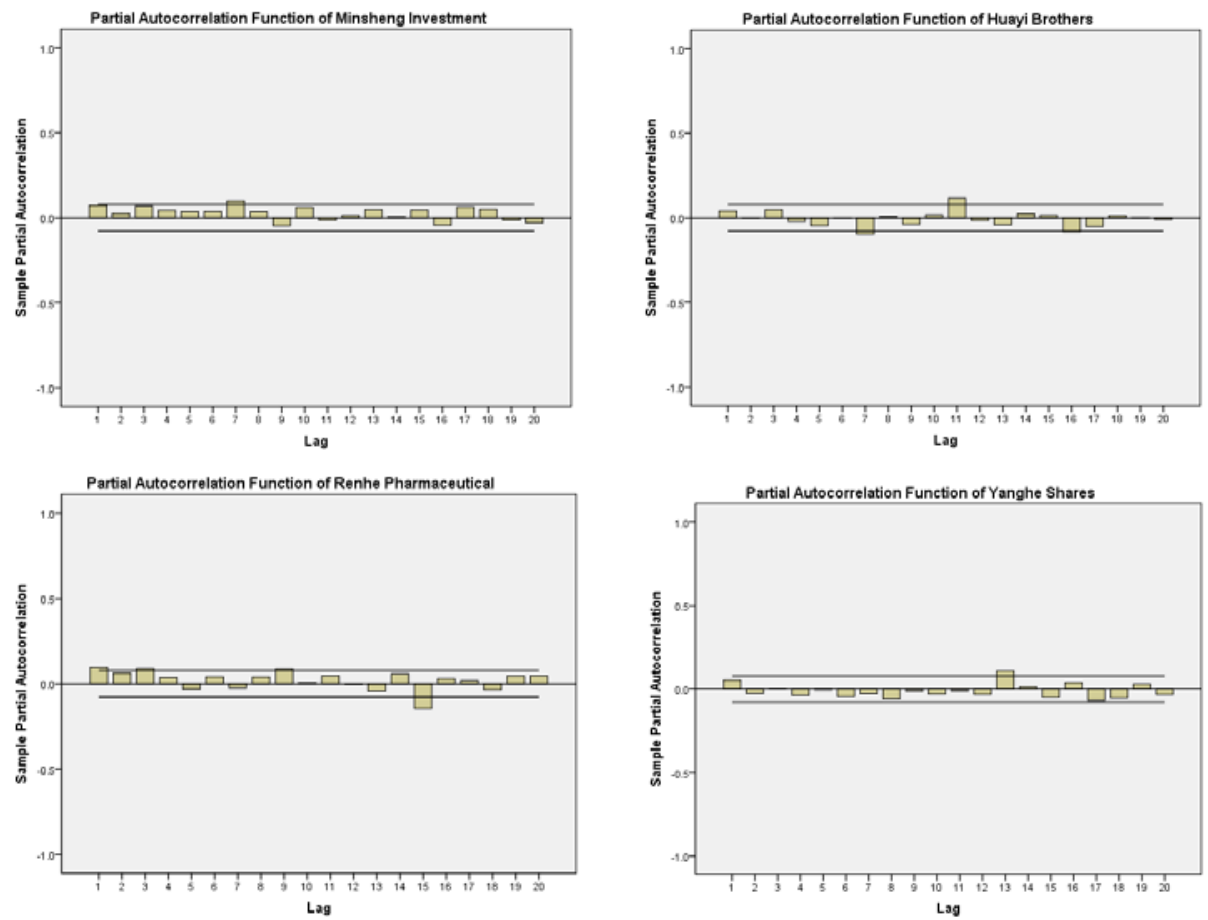

Fig.3 Partial Autocorrelation Function Images of Sample Data

According to Fig. 2 and Fig. 3, the autocorrelation function (ACF) and the partial autocorrelation function (PACF) of the sample data do not have significant correlations. In the following, we use the $\mathrm{Q}$ test and the LM test to quantitatively analyze the correlation of the sample data [3]. The data from Table 3 are derived from the econometric software Eviews8.0. The original hypothesis is that the sequence of yield is not relevant, assuming that the delay is 10,15 and 20 :

Table 3 Q test results for sample data

\begin{tabular}{c|c|c|c|c}
\hline & MI & HB & RP & YS \\
\hline P (lag=10) & 0.1683 & 0.3582 & 0.0534 & 0.7708 \\
\hline P (lag=15) & 0.0312 & 0.0803 & 0.0082 & 0.3051 \\
\hline P (lag=20) & 0.0384 & 0.1806 & 0.0134 & 0.2054 \\
\hline
\end{tabular}

When Lag $=10$, the P values of the Q test of the four stocks are greater than the significant level of 0.05 , so there is no obvious sequence correlation to the original hypothesis. When Lag $=15$ or 20 , the $\mathrm{P}$ values of Minsheng Investment and Renhe Pharmaceutical are all less than the significant level of 0.05 , so the original hypothesis is rejected and considered to have a certain series of correlations. Table 4 shows the results of LM test with $\alpha=0.05$ for the sample data: 
Table 4 LM test results

\begin{tabular}{c|c|c|c|c|c}
\hline & & MI & HB & RP & YS \\
\hline \multirow{4}{*}{ Lag=10 } & P & 0.0173 & 0.0347 & 0.0111 & 0.0279 \\
\cline { 2 - 6 } & Log likelihood & -1477.682 & -1538.149 & -1463.711 & -1490.122 \\
\cline { 2 - 6 } & AIC & 4.5907 & 4.7770 & 4.5476 & 4.6288 \\
\hline \multirow{3}{*}{ Lag=15 } & $\mathrm{P}$ & 0.0257 & 0.0321 & 0.0003 & 0.0386 \\
\cline { 2 - 6 } & Log likelihood & -1475.983 & -1532.653 & -1401.922 & -1574.947 \\
\cline { 2 - 6 } & AIC & 4.6009 & 4.7755 & 4.5336 & 4.6983 \\
\hline \multirow{3}{*}{ Lag=20 } & P & 0.0393 & 0.0401 & 0.0010 & 0.0289 \\
\cline { 2 - 6 } & Log likelihood & -1549.292 & -1589.926 & -1437.925 & -1489.042 \\
\cline { 2 - 6 } & AIC & 4.8924 & 4.9925 & 4.5812 & 5.7693 \\
\hline
\end{tabular}

The test results show that the logarithmic yield of the sample data has an ARCH effect at a significant level of $\alpha=0.05$. Therefore, we must take the appropriate way to correct the residual sequence correlation, that is, by constructing a reasonable model to describe the yield sequence [3].

\section{Calculation of VaR with Copula- GARCH Model by Monte Carlo Simulation}

Determination and Parameter Estimation of GARCH Model. Due to the large number of GARCH models, we need to select the GARCH model whose fitting effect on the sample data is the best. The sequence of yields in financial markets usually has a spike in real life, so GARCH and GARCH-Normal models are not the most reasonable choice. A large number of empirical studies have shown that, in most cases, the GARCH $(1,1)$ has been able to fully reflect the volatility of long-term financial data, and can better describe the distribution of financial time series spikes characteristic. Consider the following GARCH $(1,1)$ model:

$$
\begin{aligned}
& \sigma_{t}^{2}=\omega+\theta_{1} e_{t-1}^{2}+\theta_{2} \sigma_{t-1}^{2} \\
& R_{t}=\alpha_{0}+\alpha_{1} R_{t-1}+\beta_{1} X_{t-1}+e_{t}
\end{aligned}
$$

In this paper, the GARCH-t and GARCH-GED models are used to analyze the sample data, and all parameters are obtained by Eviews8.0. The GARCH model fitting parameters are shown in Table 5-Table 6.

Table 5 GARCH $(1,1)$-GED Parameter Estimation Results

\begin{tabular}{c|c|c|c|c}
\hline & MI (L=-37.20111) & HB (L=-1114.780) & RP $(\mathrm{L}=-922.168)$ & YS (L=-759.0163) \\
\hline$\alpha_{0}$ & 0.002120 & $2.78 \times 10^{-6}$ & $-1.22 \times 10^{-7}$ & 0.003570 \\
\hline$\alpha_{1}$ & 0.002120 & $4.96 \times 10^{-8}$ & $1.29 \times 10^{-8}$ & $6.35 \times 10^{-5}$ \\
\hline$\beta_{1}$ & 0.000394 & $-1.18 \times 10^{-7}$ & $7.08 \times 10^{-9}$ & $-6.15 \times 10^{-5}$ \\
\hline$\omega$ & 7.757033 & 1509.833 & 331.6635 & 3.975973 \\
\hline$\theta_{1}$ & 6.119045 & 7616.392 & 655.8124 & 1.382086 \\
\hline$\theta_{2}$ & -0.011284 & 1.015719 & 1.016167 & 0.992929 \\
\hline AIC & 0.136423 & -3.419075 & -1.911014 & 2.364248 \\
\hline
\end{tabular}

Table 6 GARCH $(1,1)$-t Parameter Estimation Results

\begin{tabular}{c|c|c|c|c}
\hline & MI $(\mathrm{L}=-550.8179)$ & HB $(\mathrm{L}=-1185.191)$ & $\mathrm{RP}(\mathrm{L}=-984.6357)$ & $\mathrm{YS}(\mathrm{L}=-1351.976)$ \\
\hline$\alpha_{0}$ & $4.20 \times 10^{-5}$ & -0.013523 & 0.099602 & -0.032553 \\
\hline$\alpha_{1}$ & 0.034825 & -0.074412 & -0.044314 & -0.044949 \\
\hline$\beta_{1}$ & $-3.18 \times 10^{-6}$ & 0.000577 & -0.005794 & 0.000905 \\
\hline$\omega$ & $2.26 \times 10^{-5}$ & 0.000241 & 0.004683 & 257.3169 \\
\hline$\theta_{1}$ & 24.57436 & 4.083217 & 5.177080 & 85.13800 \\
\hline$\theta_{2}$ & 0.243765 & 0.669772 & 0.672916 & 0.933637 \\
\hline AIC & 1.721660 & 3.679603 & 3.060604 & 4.194372 \\
\hline
\end{tabular}


The following criteria are followed when selecting the GARCH model:

1. The P values of all the parameters in the GARCH model are less than 0.05;

2. On the basis of 1 , the likelihood function value $(\mathrm{L})$ is chosen to be the largest, while the AIC minimum GARCH model, because the value of AIC contains the estimated value of the model and the parameters of the data, the smaller the AIC value, the better the model [5].

Therefore, according to the above selection criteria, GARCH $(1,1)$-GED can better describe the fluctuation of each index yield sequence.

Estimation and Verification of Parameters of Copula Function. The choice of Copula function does not have a standard of rule, and this paper selects Gaussian-Copula function to establish the model. The results of the correlation of the Gaussian-Copula function are shown in Table 7, and the results are from MATLAB 2016a.

Table 7 Estimation of Linear Correlation Parameters Matrix for Gaussian - Copula Functions

\begin{tabular}{c|c|c|c|c}
\hline Sample & MI & HB & RP & YS \\
\hline MI & 1.0000 & -0.1616 & 0.2431 & 0.1274 \\
\hline HB & -0.1616 & 1.0000 & -0.1936 & 0.0293 \\
\hline RP & 0.2431 & -0.1936 & 1.0000 & 0.1994 \\
\hline YS & 0.1274 & 0.0293 & 0.1994 & 1.0000 \\
\hline
\end{tabular}

According to the data in Table 7, the correlation between the individual stock price series is very small, so we can see that the choice of different stocks for the portfolio, to a certain extent, can reduce the risk.

The K-S test is a nonparametric test method that is often used to test whether a particular sample is subject to a particular distribution. The results of the K-S test are shown in Table 8, and the results are from IBM SPSS Statistics 21.

Table 8 K-S test results

\begin{tabular}{c|c|c|c|c}
\hline Sample & MI & HB & RP & YS \\
\hline K-S Value & 0.2367 & 0.2105 & 0.2182 & 0.1750 \\
\hline P Value & 0.8179 & 0.4464 & 0.7130 & 0.6762 \\
\hline
\end{tabular}

The probability of K-S test in Table 8 is greater than 0.6 , the greater the probability that the better the model fitting effect, that is to say the established Copula-GARCH model can well describe the distribution characteristics of the stock.

Simulation of VaR values. After the Copula-GARCH model being built and tested, the VaR values can be calculated and the defects in the traditional VaR calculations can be optimized. In this paper, Monte Carlo simulation is used to calculate VaR.

The optimal portfolio weight coefficients $\left(\mu_{1}, \mu_{2}, \mu_{3}, \mu_{4}\right)$ and the corresponding minimum VaR values at different confidence levels in Table 9 are obtained according to the above method.

Table 9 Optimal Portfolio Weight Coefficient under Different Confidence Levels

\begin{tabular}{c|c|c|c|c|c|c|c}
\hline Serial Number & $1-\alpha$ & $\min \left(\mathrm{VaR}_{\alpha}\right)$ & Combined Return & $\mathrm{MI}\left(\mu_{1}\right)$ & $\mathrm{HB}\left(\mu_{1}\right)$ & $\mathrm{RP}\left(\mu_{1}\right)$ & $\mathrm{YS}\left(\mu_{1}\right)$ \\
\hline 1 & $90 \%$ & -0.0374 & 0.0373 & 0.2594 & 0.2915 & 0.2625 & 0.1867 \\
\hline 2 & $91 \%$ & -0.0381 & 0.0444 & 0.2685 & 0.2458 & 0.2963 & 0.1895 \\
\hline 3 & $92 \%$ & -0.0399 & 0.0516 & 0.2776 & 0.2000 & 0.3301 & 0.1922 \\
\hline 4 & $93 \%$ & -0.0418 & 0.0588 & 0.2868 & 0.1542 & 0.3640 & 0.1950 \\
\hline 5 & $94 \%$ & -0.0440 & 0.0660 & 0.2959 & 0.1085 & 0.3978 & 0.1978 \\
\hline 6 & $95 \%$ & -0.0465 & 0.0732 & 0.3050 & 0.0627 & 0.4316 & 0.2006 \\
\hline 7 & $96 \%$ & -0.0491 & 0.0803 & 0.3141 & 0.0170 & 0.4655 & 0.2034 \\
\hline 8 & $97 \%$ & -0.0526 & 0.0875 & 0.2592 & 0 & 0.5992 & 0.1416 \\
\hline 9 & $98 \%$ & -0.0573 & 0.0947 & 0.1666 & 0 & 0.7919 & 0.0415 \\
\hline 10 & $99 \%$ & -0.0649 & 0.1019 & 0 & 0 & 1.0000 & 0 \\
\hline
\end{tabular}


The results in Table 9 show that, except for the confidence rate of $97 \%$ to $99 \%$, the minimum risk portfolio under different confidence in the weight coefficient is not very different, and the investment is mainly concentrated in the Renhe Pharmaceutical and Minsheng Investment. It is because the yield of Minsheng Investment in the sample timing shows an upward trend, and Renhe Pharmaceutical's yield fluctuations is relatively stable. In the following, we perform the Kupiec test on the VaR calculated by the Copula-GARCH model.

Table 10 Kupiec test results

\begin{tabular}{c|c|c|c}
\hline$\alpha$ & VaR & LR-Value & Acceptable interval \\
\hline 0.01 & 6.4906 & 8.39228 & $2 \leq \mathrm{N} \leq 14$ \\
\hline 0.02 & 5.7305 & 5.67231 & $6 \leq \mathrm{N} \leq 22$ \\
\hline 0.05 & 4.6550 & 3.31859 & $23 \leq \mathrm{N} \leq 42$ \\
\hline 0.1 & 3.7409 & 1.44898 & $55 \leq \mathrm{N} \leq 75$ \\
\hline
\end{tabular}

From the results of the failure rate test in Table 10, it can be seen that the LR value is less than the $\chi^{2}$ value for a given significance level. So we accept the original hypothesis that the Copula-GARCH model is valid.

\section{Conclusions}

For portfolio risk management, it is particularly important to portray the relevant structure between financial assets. In this paper, the GARCH model and the Copula function are used to study the risk value of the logarithmic yield of four stocks belonging to different industries, and the following conclusions can be obtained:

1. The data samples of the logarithmic yield of the four stocks are far from the normal distribution, indicating that they all have spikes. The traditional GARCH model is fitted with the assumption that the data is subject to a normal distribution, so this paper selects the GARCH-GED model to simulate the edge distribution;

2. For the estimation of the edge distribution, if we use EGARCH to improve the model, we can explain the bad and good news on the impact of asymmetry and other phenomena, which may make the analysis results more practical significance [6].

3. For the improved Copula distribution estimation algorithm, although the calculation results are better than the previous algorithm, the results change little, indicating that there is the possibility of further improvement.

\section{References}

[1] Yanhua Wei, Shiying Zhang: Copula Theory and Its Application in Financial Analysis. Peking: Tsinghua University Press, 2008.

[2] Information on http://www1.resset.cn:8080/product/common/main.jsp

[3] Dawei Liu: Portfolio Risk Analysis Based on Copula-GARCH. Tianjin: Tianjin University of Science and Technology Press (2006).

[4] Yijue Bao: An Analysis of Portfolio VaR Value - Based on Copula's Theory.Dalian: Northeast University of Finance and Economics Press (2012).

[5] Shupeng Li: Research on Portfolio Risk Based on GARCH - GPD - COPULA Function. Tianjin: Tianjin University of Finance and Economics Press (2012).

[6] Zhenxiang Wu, Min Chen, Wuyi Ye and Boqi Liao, in: Portfolio Risk Analysis Based on Copula-GARCH, volume 3 of System Engineering Theory and Practice (2006.03). 